\title{
A High-Bandwidth Fiber-Optic System Using Laser-Diode Transmitters
}

\author{
B. L. Pruett, F. Roeske, R. A. Jones, and K. G. Hagans \\ Lawrence Livermore National Laboratory \\ P. O. Box 808, Mail Stop L-45 \\ Livermore, California 94550 \\ T. J. Davies and M. A. Nelson \\ EG\&G/Santa Barbara Operations \\ 130 Robin Hill Road \\ Goleta, California 93017 \\ G. C. Gong \\ Mitre Corporation \\ Burlington Road \\ Bedford, Massacliusetts 01730
}

This paper was prepared for the Proceedings of the Society of Photo-Optical Instrumentation Engineers August 19-23, 1985

San Diego, California

July 1985

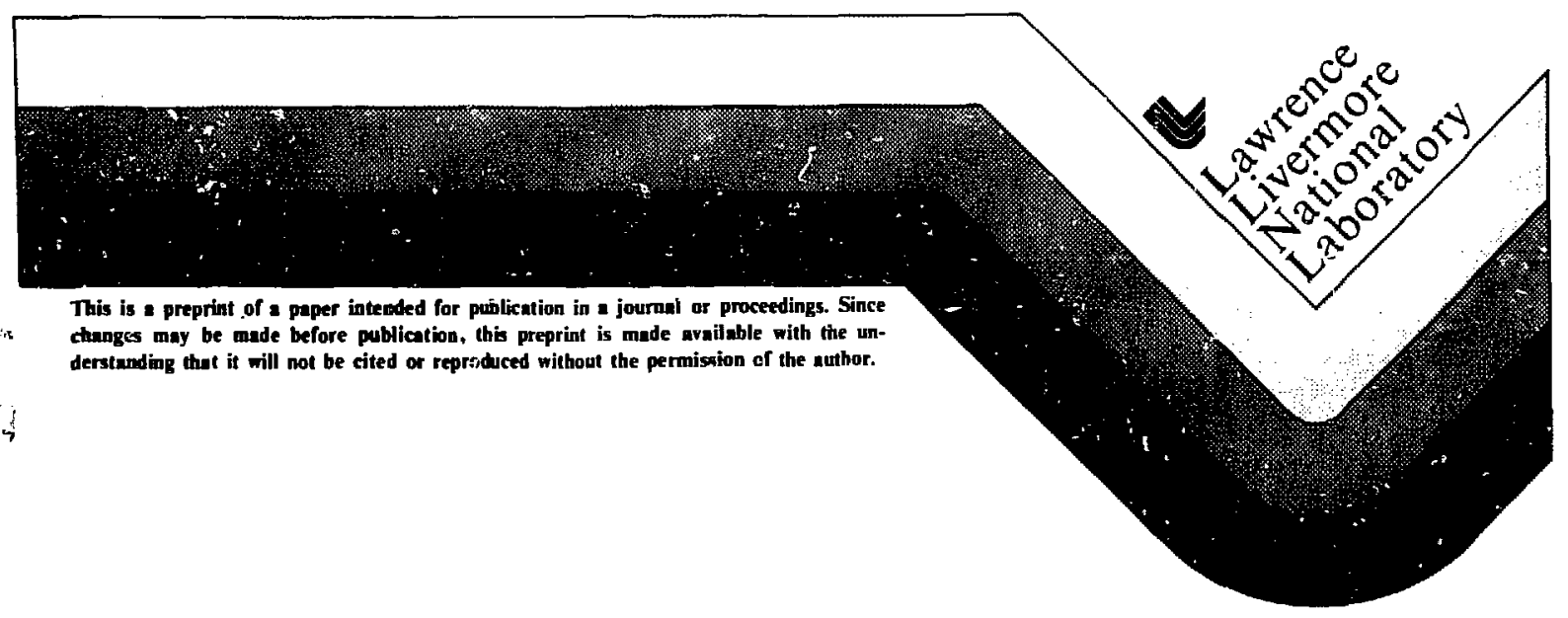




\title{
A High-Bandwidth Fiber-Optic System Using Laser-Diode Transmitters
}

\begin{abstract}
A system has been developed to transmit analog sig , als through 1-km-long fiber-optic cables having bandwidths up to $1.5 \mathrm{GHz}$. Semiconductor injection laser-diade transmitters operating at a wavelength of about $815 \mathrm{~nm}$ are directly modulated by electrical signals frum radiation detectors. Graded-index optical fibers transmit the optical signals to a remote facility, where they are recorded on a single streak camera.
\end{abstract}

\section{Introduction}

A diagnostics system that uses fiber optics has been developed to measure radiation produced by an underground nuclear explosion. Designed to transmit and record analog signals of about $1 \mathrm{GHz}$ through fibers up to $\mathbf{1} \mathbf{k m}$ in length, the system has to tolerate the difficult conditions associated with the preparation and execution of an underground experiment, including stress and abrasion on cables, high temperatures, and intense radiation backgrounds.

In the traditional diagnostics systems, radiation detectors are used to obtain electrical signals, which are transmitted by coaxial cables to a surface oscilloscope-recording facility. In the new optical-fiber system, the electrical signals from radiation detectors are used to directly modulate a laser-diode transmitter, and the resultant optical signals are sent through optical fibers to be recorded by a streak-tamera receiver.
Advantages of the optical-fiber system, as compared with coaxial-cable systems, include:

- High bandpass. The optical-fiber system has a bandpass up to $1.5 \mathrm{GHz}$ with a $1-\mathrm{km}$ transmission path length, as compared with about $0.4 \mathrm{GHz}$ for the coaxial cable system.

- High sensitivity. Sensitivity is about 100 times greater, which greatly increases the usable dynamic range of the detectors and can reduce the number required.

- Multichannel recording. More than 20 data channels can be recorded on a single streak camera, facilitating interchannel timing and amplitude comparisons and significantly reducing the space and manpower required by oscilloscope recording.

\section{System Description}

The fiber-optic diagnostics system consists of a radiation detector, a laser-diode transmitter, an optical-fiber cable, and a streak-camera receiver (Fig. 1). A gamma-ray diagnostics system using the same fiber cable and streak camera has been previcusly reported. ${ }^{1}$

\section{Radiation Detector}

The radiation detector for this system can be any of those in the suite of detectors used with the cuaxial-cable systems. These detectors typically have very high linear-current capabilities (up to $200 \mathrm{~A}$ ), while the transmitters have maximum input currents of less than $0.2 \mathrm{~A}$. Consequently, one detector can drive several transmitters to cover a large dynamic range by using an appropriate combination of tees and attenuators.

The detectors have a bandpass as high as $2 \mathrm{GHz}$. Those that are marginal for a 1.0- to 1.5$\mathrm{GHz}$ system con probably be redesigned for increased bandwidth, but at the expense of reduced sensitivity and reduced maximum linear current.

\section{Laser-Diode Transmitter}

The transmitter consists of a single longitudinalmode injection laser diode, a power supply, regulation circuitry, and a bias tee input circuit 

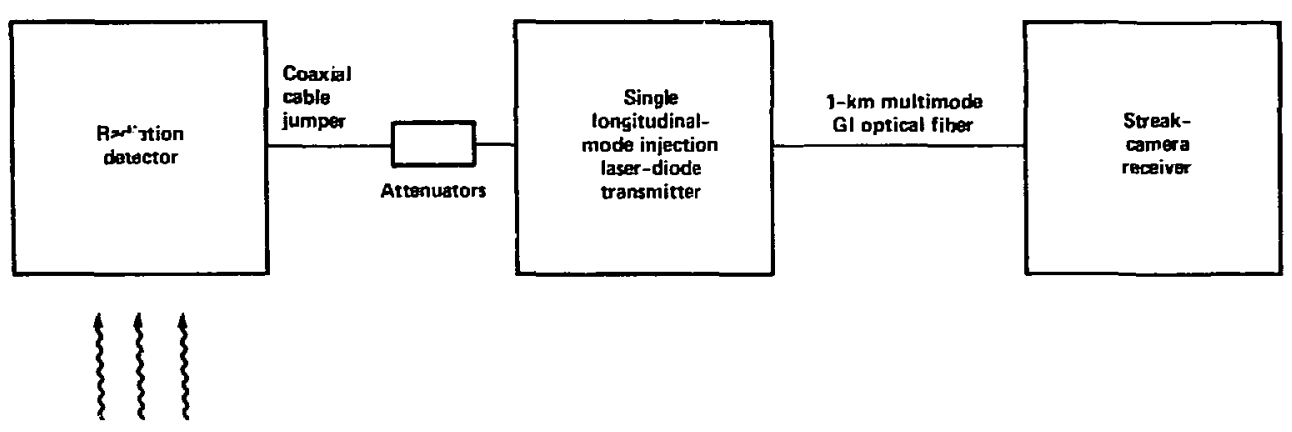

Figure 1. Basic arrangement of the laser-diode fiber-optic diagnostics system.

(Fig. 2). The laser diodes used to date have been the Ortel Corporation models LDS10-PM and LDS10-PMF; typical characteristics of these diodes are summarized in Table 1. A typical response of light output vs current input is shown in Fig. 3.

For positive-polarity signals, typical of most of the detectors used, the laser is biased just above lasing threshold at the bottom of the linearresponse region. The dc bias level is applied to the low-pass side of the bias tee. A photodiode detector in the laser package senses the continuous optical power level and is used by the regulation circuit to keep the power constant. The input signal is applied to the high-pass side of the bias tee.

The bias level must be increased when a bipolar input signal is to be accommodated, and a pulse bias scheme is used to prevent the input of excessive power to the receiver for a long time period. With the transmitter operating with the bias near the lasing threshold level, as described above, a long rectangular pulse is mixed into the signal input absut $1 \mu \mathrm{s}$ prior to the expected event time. The pulse amplitude is selected to permit the full range of negative and positive excursions of the expected signal. For negative signals, either a high-frequency inverting transformer or the pulse bias can be used.

\section{Optical-Fiber Cable}

Signals are carried from the transmitter to the receiver by a $50-\mu \mathrm{m}$-diameter graded-index (GI) fiber. Each fiber has a numerical aperture of about 0.2 , an attenuation of about $2 \mathrm{~dB} / \mathrm{km}$, and can transmit frequencies up to $1.5 \mathrm{GHz}$ for fibers approaching $1 \mathrm{~km}$ in length. The optical fibers are

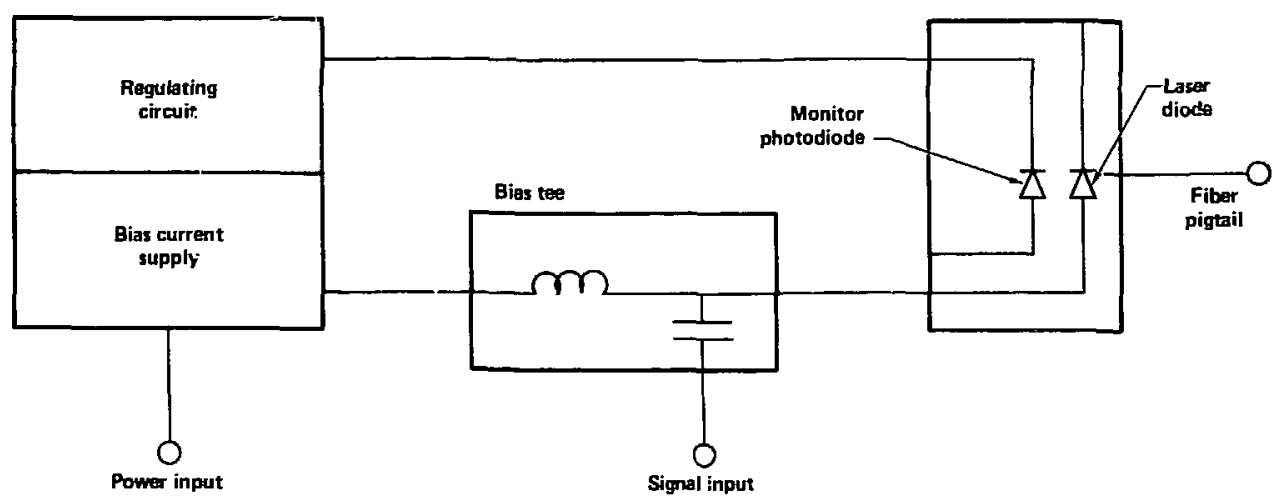

Figure 2. Components in the transmitter package. 
Table 1. Typical characteristics of the GaAlAs laser diodes used in the transmitter.

\begin{tabular}{|lcc|}
\hline \multicolumn{1}{|c|}{ Type } & LDS10-PM & LDS10-PMF \\
\hline Modulation bandwidth (GHz) & 3 & 6 \\
Input polarity & Positive & Positive \\
Input impedance (D) & 50 & 50 \\
Threshold current (mA) & 20 & 12 \\
Maximum forward voltage (V) & 3.6 & 3.2 \\
Wavelength (nm) & 817 & B17 \\
Maximum fiber pigtail power (mW) & 3.5 & 3.5 \\
Operating lemperahure ('C) & 0 to 50 & 0 \$0 50 \\
\hline
\end{tabular}

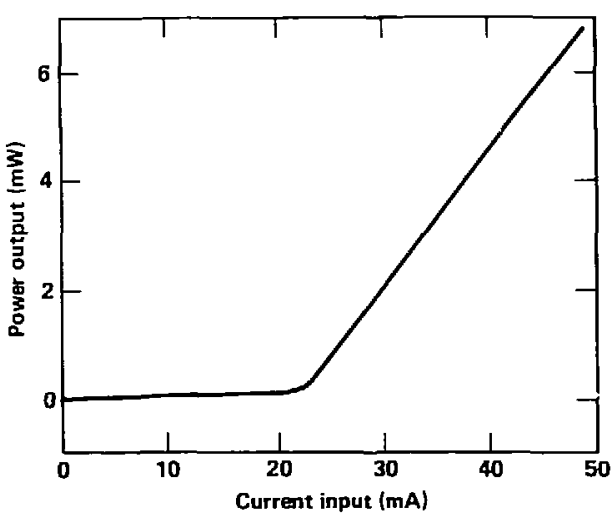

Figure 3. Typical response for an injection laser diode used in the transmitter.

placed in specially fabricated nonmetallic cables to provide protection in both the surface and downhole runs and to prevent the transmission of radioactive gas from the underground explosion to the surface.

\section{Streak-Camera Receiver}

The primary receiver is a sireak camera developed at Lawrence Livermore National Laboratory (LLNL). For some applications, photomultiplier detectors and oscilloscopes have been used instead of a streak camera.

The streak camera uses an RCA streak tube and an IT\&T 40-mm-riam microchannel-plate intensifier between the streak tube phosphor and the film. The data are recorded on Kodak Royal- $X$ Pan film. The sweep time is normally $60 \mathrm{~ns}$, but it can be changed to $15 \mathrm{~ns}$ for better time resolution.

The 60-ns camera has a response time of less than $0.2 \mathrm{~ns}$. At the operating wavelengths of the transmitters, the camera will record from less than $10 \mu \mathrm{W}$ to more than $1 \mathrm{~mW}$. As many as 25 information channels can be recorded simultaneously.

\section{System Calibration}

System calibrations include interchannel timing and amplitude calibrations.

\section{Interchannel Timing}

Analyses of nuclear-test data require precise measurement of the relative times of the signals produced in the different channels. These measurements can be affected by either mechanical stretching or thermal expansion of the fiber cables during emplacement of the diagnostics-canister assembly.

Any emplacement effect can be determined by injecting a fast-rise-time electrical test pulse simultaneously into each channel and recording the pulses with the streak camera (Fig. 4). Relative timing is determined to less than $1 \mathrm{~ns}$ by this technique.

\section{Amplitude Calibration}

Environmental factors can also affect the amplitude calibration of the diagnostics system. Individual components and connections are monitored prior to and during installation into tise system, and, after installation, both dc and pulse calibrations are done on all or part of the system.

Once the dc bias-level optical output of a transmitter has been set, it should remain constant when there is no electrical signal applied. This 




Figure 4. Streak-camera record for a typical measurement. The top trace is a time base with a series of short pulses applied at 1-ns intervals. The second trace is a time reference mark. The lower four traces are diagnostic data channels, with a simultaneity signal appearing near the center of each trace.

level is monitored as a convenient measure both of system integrity and of any changes that might occur during the emplacement operation.
Dynamic calibrations can be obtained by using pulsed electrical inputs characteristic of the expected nuclear test signals.

\section{Data Analysis}

Film records from the streak camera (Fig. 4) contain the signal data in density-vs-position format. Time-base traces, consisting of fast repetitive pulses, and other time marks are used for reference to other diagnostics systems. Separately, a density-vs-exposure calibration wedge is placed on the film prior to development.

The data are reduced in 10 steps, according to the following sequence:

1. The film record is digitized to give density-vsposition data.

2. Position data are converted to time data using the time base.

3. Film-density values are converted to filmexposure values by using the calibration wedge.

4. The data are smoothed to remove fluctuatior is (caused mostly by film structure).
5. The film fog-level eqquivalent exposure value is subtracteci from the data.

6. The streak-camera transfer function is used to convert the data from exposure vs time to streak-camera power input vs time.

7. For each channel, the laser-transmitter biaslevel optical power, as ohserved prior to signal arrival, is subtracted from the data.

8. For certain measurements, a system timeresponse function is removed from the data by using a deconvolution program.

9. The streak-camera power input-vs-time data are converted to the proper signal-input ampliude units-vs-time data by using the known sensitivity and transmission values for each component.

10. The time coordinates are adjusted to a desired reference by using the time-mark data.

\section{System Performance}

The time response of the system with a $1-\mathrm{km}$-long optical fiber and a $60 \mathrm{~ns}$ streak camera has been measured using a 0.2-ns-wide electrical input pulse. Figure 5 shows this pulse and the corresponding output data. The data have been filtered mathematically with a 3-GHz low-pass filter to help remove fluctuations caused by the film. Lower-frequency film-induced fluctuations, as seen in the baseline, are maintained. The output- pulse risetime is about 0.24 ns, and the FWHM is about $0.41 \mathrm{~ns}$.

Dynamic range and signal-to-noise ratio for this system have been found to largely depend upon the film "noise" problem and the level of film exposure. For optimum conditions, a dynamic range in excess of $16 \mathrm{~dB}$ (optical power) has been obtained for each channel. 

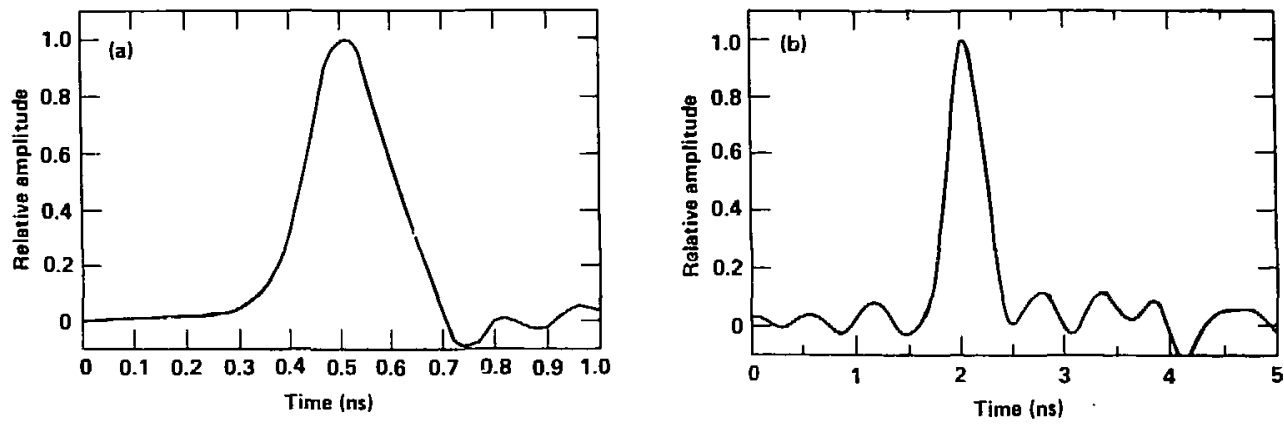

Figure 5. Measurement of the system time response. (a) A 0.2-ns-wide pulse applied to the transmitter input. (b) A 0.4-ns-wic' pulse obtained from the streak-camera record.

\section{Future Improvements}

Work is in progress to extend the dynamic range, frequency response, and accuracy of this fiberoptic diagnostics system. The use of a finer-grain recording film and different developing techniques should lead to both significantly inproved data and an increase in dynamic range and accuracy. Solid-state electronic-readout cameras will also be investigated to obtain further improvements.

A similar diagnostics system using a singlemode fiber cable is under consideration. This altemative system, which probably would use a $10-\mathrm{GHz}$ transmitter and a streak camera with a 15-ns sweep length, is expected to provide a time response in the range of several $\mathrm{GHz}$. Efforts have been started on the development of radiation detectors that would be compatible with transmission and recording systems operating in this high-frequency range.

\section{Acknowledgments}

We wish to recognize the assistance provided to this effort by R. T. Peterson, D. E. Smith, J. M. Ticehurst, C. H. Dittmore, and C. G. Neeld, all at LLNL; and by T. Pagano and F. Janson, both at EG\&G/Santa Barbara.

This work was performed under the auspices of the U.S. Department of Enery by the Lawrence Livermore National Laboratory under contract No. W-7405-ENG-48.

\section{Reference}

1. F. Roeske, D. E. Smith, B. L. Pruett, and R. P. Reedy, "A High-Bandwidth Multichannel Fiker Optic System for Measuring Gamma Rays," Proc. SPIE Ann. Tech. Sympos., 1984 (SPIE, San Diego, CA, Aug. 19, 1984), vol. 506, p. 29. 